\title{
OxIS 2019: Dueling Perspectives on the Internet in Britain
}

by Grant Blank and William H. Dutton with assistance from Julia Lefkowitz

A report on the 2019 Oxford Internet Survey, Oxford Internet Institute, University of Oxford. The authors thank the UK Department for Digital, Culture, Media \& Sport; Google Inc., and BT, for their support of this survey. This report introduces a series of reports on OxIS 2019.

Britain celebrates Alan Turing, a pioneer in shaping early computer technology, such as by choosing his image for the nation's fifty-pound note. And the nation regularly honors Sir Tim Berners-Lee, whose team at CERN invented the World Wide Web. With half the world online, no nation wants its population to be on the wrong side of the digital divide. Not surprisingly, Britain, in line with many nations, continues efforts to build fiber infrastructure to bring high-speed broadband and wireless connectivity to all ${ }^{1}$ and catch up with other leading industrial nations. ${ }^{2}$

At the same time, in the same nation, and around the world, it is clear that media pundits, politicians ${ }^{3}$, and leading academics ${ }^{4}$ are raising a growing cascade of dystopian alarms over the cyber harms attributed to screen time, social media, disinformation, and malicious content online. In the aftermath of surprising election results such as the UK's 2016 vote to leave the EU and the 2016 Presidential elections in the USA, the press and other major institutions have joined a virtual 'tech-lash' against the big Internet companies that are increasingly seen as threats to privacy, democracy, and society. Despite research challenging many claims about the role of the Internet and social media, dramatic media portrayals, such as The Great Hack, have captured the public's imagination in ways that parallel Vance

${ }^{1}$ BT present fibre strategy to the UK government:

https://www.ft.com/content/2fafb366-b4f3-11e9-8cb2-799a3a8cf37b

2 See World Internet Statistics: https://www.statista.com/topics/1145/internet-usageworldwide/

${ }^{3}$ For example, see the Government's April 2019 Cyber Harms White Paper: https://assets.publishing.service.gov.uk/government/uploads/system/uploads/attach ment data/file/793360/Online Harms White Paper.pdf

${ }^{4}$ Manuel Castells, M. (2019), 'Foreword' to Graham, M., and Dutton, W. H. (eds), Society and the Internet, $2^{\text {nd }}$ Edition. Oxford: Oxford University Press. 
Packard's (1957) portrayal of advertising in the age of broadcasting in his The Hidden Persuaders. ${ }^{5}$ One leading British journalist has blamed social media for a 'national nervous breakdown'.6

Which world is more in line with the realities of life, work, and politics online in 2019 Britain? Has the optimism of the early $21^{\text {st }}$ century given way to dystopian realities of the online world? How can such competing perspectives be reconciled?

This blog is designed to build on and complement the initial summary of OxIS 2019 findings. ${ }^{7}$ We argue that the realities of Internet use in Britain raise serious questions for the dystopian critics. Despite a growing chorus of voices on the threats posed to our lives by the Internet and social media, it is clear that the views, behavior, and habits of Internet users in Britain indicate that they continue to embed the Internet and social media more closely in more aspects of their everyday lives. Internet users are finding the technologies and services they provide to be more valuable for their access to information and entertainment in myriad ways, while mainstream, traditional media are increasingly losing their hold on our habits and ways of life. Should these findings deepen or reduce concerns over the threats posed by the Internet and social media? We believe they challenge many aspects of the dystopian rhetoric about the Internet of today.

In this brief introductory blog, we will describe the pattern of key findings behind this general conclusion. Following blogs will fill in more of the detail behind nine basic trends that lead us to suggest a more positive view of the Internet's role in everyday life as well as the state of democracy. All these trends are based on the 2019 Oxford Internet Survey (OxIS), which was supported through funding from the UK's Department of Digital, Culture, Media \& Sport (DCMS), BT, and Google Inc. The survey was designed, administered, and interpreted by the authors, who take full

\footnotetext{
${ }^{5}$ Vance Packard (1957), The Hidden Persuaders. New York: David McKay Company, Inc.

6 Tim Lott, 2019, 'What Caused Britain's National Nervous Breakdown?', The Guardian, 16 July: https://www.theguardian.com/commentisfree/2019/jul/16/britainnational-nervous-breakdown-internet-social-media

7 https://oxis.oii.ox.ac.uk/wp-content/uploads/sites/43/2019/09/OxIS-report-2019final-digital-PDFA.pdf
} 
responsibility for the findings, which do not necessarily reflect the views or interpretations of the OII, or any of the organizations that have supported this research.

The Oxford Internet Survey (OxIS) 2019

The 2019 Oxford Internet Survey (OxIS) provides a snapshot of how individuals view and use the Internet, social media, and related information and communication technologies (ICTs) in Britain. It builds on earlier surveys that have been done biannually from 2003 to 2013 by the Oxford Internet Institute (OII) to capture how people use or don't use the Internet, and continuities and changes in how its use and implications have changed overtime. ${ }^{8}$ It addresses straightforward questions: Who uses the Internet and related digital media? What are people in Britain doing online pursuing entertainment, information, killing time? How do they view the Internet, including its opportunities and risks? Why are some people not using the Internet, and why do many users employ the Internet in more limited ways than others? What difference does the Internet and social media make in their lives?

Nine Findings to Take Away from the 2019 Survey

Given such a widespread and influential consensus on the value and opportunities opened up online, the rise of dystopian narratives about the threats and harms attributable to the Internet created a need to revisit the questions OxIS has been asking over earlier years. It is more critical than in earlier years to ask whether people in Britain are retreating from their growing relationship to the online world. This report enables us to see continuities and changes over the last six years, from 2013-19. While the overall pattern conflicts with the dystopian narrative of growing harms, the findings are remarkable in multiple respects. Consider the following:

1. Divides. Diffusion has slowed, narrowing the digital divide, but at the same time it has been deepened. It has narrowed from a continuing diffusion of the Internet although its spread has slowed, leaving a prominent digital divide

\footnotetext{
${ }^{8}$ Information about the OxIS surveys is available here: https://oxis.oil.ox.ac.uk
} 
prominent in Britain, with more than 15 percent of the public off line.

Nevertheless, a larger proportion of the public in Britain use the Internet in 2019 than in 2013, despite disappointing gains for those seeking to address inequalities in online access. However, the divide has deepened in that some people have gained greater access to the Internet via more devices, some of which are mobile. In 2011, we referred to a new generation of users - who we called 'next generation users' - who are more accessible online anywhere at any time. Additional devices have deepened the divide between Internet users and non-users, who have no or limited access and those with multiple forms of access to the online world. ${ }^{9}$ Next generation users tend to be the most engaged users who have integrated the Internet most fully into all parts of their lives. And the ranks of these Next General Users have grown substantially over the last six years. Nevertheless, the prominence of scary images of the dystopian role of the Internet are likely to keep many non-users offline, especially since experience with the Internet has proven to be one of the most important ways to gain the public's trust and confidence in its value. ${ }^{10}$

2. Mobiles. There has been dramatic change in how people use the Internet, primarily defined by a sharp and continuing rise in the number of ways people use mobile Internet - primarily on smartphones - to perform many functions previously served by computers and laptops. This rise has enabled people to more closely embed the Internet in their everyday lives, such as through making payments and banking on their mobile.

3. Habits. Most generally, the Internet has become more deeply embedded and routinized as it defines how we do what we do. Despite years of panic over the harms and risks posed by the Internet and social media, the Internet today is far more ingrained in everyday life. Internet users are no more concerned about their safety on the Internet than they were six years ago. To the

\footnotetext{
${ }^{9}$ Dutton, W. H., and Blank, G. (2011), Next Generation Users: The Oxford Internet Survey 2011. Oxford: Oxford Internet Institute, University of Oxford.

${ }^{10}$ Evidence of the Internet as an experience technology is supported in a number of OxIS publications, including: Blank, G., and Dutton, W. H. (2011), 'Age and Trust in the Internet: The Centrality of Experience and Attitudes Toward Technology in Britain', Social Science Computer Review, 30(2): 135-151. http://ssc.sagepub.com/content/early/2011/02/21/0894439310396186
} 
contrary, it has become so central that there are repeated debates about how people could cope without the Internet. ${ }^{11}$

4. Media. The traditional mass media of radio, television, and film are becoming visibly less mainstream. There has been a remarkable decline in the role of television, for example. The decline of legacy television, radio, and news media has been a topic of concern even before the twenty-first century, but this decline is increasingly more visible in OxIS research, with TV and radio being viewed as less essential for obtaining information albeit more important for entertainment. That said, 19 percent of the public see TV, as compared with 25 percent who view the Internet, as 'essential' for entertainment. Moreover, Internet users tend to see the Internet as comparable to mainstream media in terms of reliability and trustworthiness, particularly when accessed through the lens of search engines. ${ }^{12}$ Social media enjoys less trust and is attributed less confidence that the Internet per se or mainstream news, for example, but the public's use of social media has risen despite a constant barrage of negative anecdotes and scare stories. ${ }^{13}$

5. Disinformation. From the perspective of Internet users, the panic over fake news, echo chambers and filter bubbles has been exaggerated. While too many Internet users may selectively view or read information about politics with which they agree, they are exposed to multiple sources - more than ever before available to the public - via the Internet, search, and social media, in addition to print media, radio, and TV. Most Internet users are aware of concerns over echo chambers, filter bubbles, and disinformation, but

${ }^{11}$ For example, there is an engaging BBC treatment of what would happen if the Internet stopped working for even a day, see:

http://www.bbc.com/future/story/20170207-what-if-the-internet-stopped-for-a-day 12 Also see our related study of how people gain access to information about politics across seven nations: Dutton, W. H., Reisdorf, B. C., Blank, G., Dubois, E., and Fernandez, L. (2019), 'The Internet and Access to Information About Politics: Searching Through Filter Bubbles, Echo Chambers, and Disinformation', pp. 228247 in Graham, M., and Dutton, W. H. (eds), Society and the Internet: How Networks of Information and Communication are Changing our Lives, $2^{\text {nd }}$ Edition. Oxford: Oxford University Press.

${ }^{13}$ The 2019 OxIS findings support and reinforce the findings of the authors and a larger team on the Quello Search Project, which found the concerns over filter bubbles and echo chambers to be exaggerated, given the practices of users (Dutton et al, 2019). 
generally trust the Internet and search, although they have a healthy skepticism about the information they find on social media. That said, they are not in a state of panic. It could be useful to place more emphasis on media literacy, encouraging individuals to seek out and critically consider opinions and viewpoints that are contrary to their own views, but the media habits of most users expose them to many sources and viewpoints by virtue of multiple search algorithms and social media making diverse recommendations of relevant content.

6. Targeting. Internet users do not like targeted advertising. The public has long found tele-marketing to be a problem, and it is not surprising that they are equally if not more frustrated with the Internet being used to reach them with advertising in even more targeted ways. At the same time, the ability of some providers to target users with information of interest to them is valued by Internet users and could well be critical to the future success of traditional media outlets, such as local news sources, that do not have a mass appeal.

7. Privacy. A sizeable proportion of the public are concerned over the impact of the Internet on their privacy. However, levels of concern have not increased over the past six years. For example, reported privacy violations, such as financial fraud, have not increased in the past six years. Reported risks seem stable and this suggests that the stability of concerns is realistic. Moreover, non-users are more concerned over privacy online than are Internet users, suggesting that actual experience online tends to alleviate some of the fears central to the dystopian rhetoric around the Internet. Yet, the alarms raised over privacy might well be reinforcing the digital choice of non-users to stay offline and thereby putting themselves at a disadvantage in a digital world.

8. Cultures. All Internet users do not respond to the potential benefits and harms associated with the Internet in the same ways. As we discovered in 2013, Internet users fall into distinctly different cultures, which vary in how individuals experience and use the Internet. ${ }^{14}$ Nevertheless, some risks, such as a loss of one's privacy, breaches of security, and other cyber harms are clearly evident among Internet users in Britain. Surprisingly, however, these

${ }^{14}$ Dutton, W. H., and Blank, G. with the assistance of Groselj, D. (2013), Cultures of the Internet: The Internet in Britain: Oxford Internet Survey 2013 Report. Oxford Internet Institute, University of Oxford. 
concerns are not significantly greater in 2019 than they were in 2013, suggesting that much of the angst over most recent events, such as around the Cambridge Analytica scandal, has been limited primarily to media pundits, politicians, and academics, and do not characterize the experience of most Internet users. As in everyday life, people deal with risks, but there are different cultures of the Internet, that view the benefits and risks of being online in somewhat different ways. Our survey enables us to explore those Internet cultures - via identifiable sets of Internet users - who are most and least concerned or effected by various benefits, as well as different risks of harm.

9. Prosumers? One of the most interesting questions raised has been whether the shift to mobile and social media are reconfiguring the propensity of Internet users to create and produce online content, such as in writing a blog. The shift to mobile, and easy to click, like, and share social media, has made it easier to consume content online, on the go, and with more contacts, but has potentially made it easier to post information that is more limited to short tweets, photos, and posts, than more creative content, such as a more detailed comment or blog. Are Internet and social media users are becoming better consumers of an increasingly mass medium, or are they also producing content in ways that are likely to contribute to the promise of diversity and collective intelligence opened up by the online world.

The findings of the 2019 OxIS will be interpreted in multiple ways by different readers, but it is difficult to support the dominant dystopian narrative unless you assume that most of the public fail to understand the harms they are exposed to online. While the strong dystopian narrative can be simple and compelling news for the press and politicians, it suffers from the same degree of technological determinism that provided the logic of earlier utopian narratives about the Internet and social media. Time and again, we learn that you cannot validly extrapolate from features of a technology to predict the societal consequences of search, social media, or political advertising. We need empirical research to understand the actual uses and impacts of the Internet across different kinds of users in different social and political contexts, and a strong narrative to support the value of the Internet as it is increasingly embedded in how we do what we do in Britain and elsewhere in the 
digital world. We will be posting more focused blogs that follow up on the issues outlined above. We hope you follow our analyses and consider them in developing your own perspective on the role of the Internet in Britain and other nations. 